\title{
Evaluation of Invasive Prenatal Test Indications and Results at a Tertiary Center in the Thrace Region of Turkey
}

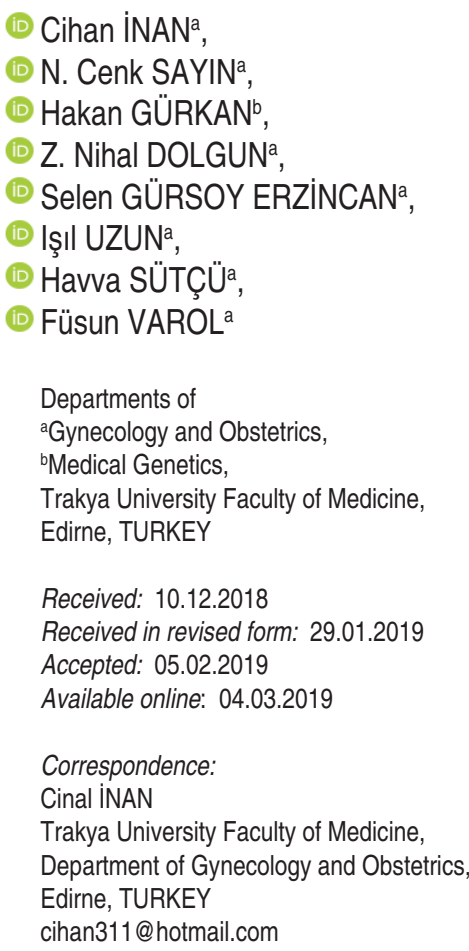

Copyright $@ 2019$ by Türkiye Klinikleri

\begin{abstract}
Objective: The present study aimed at evaluating and improving the knowledge regarding prenatal test indications by assessing the results of the invasive prenatal tests for the detection of fetal chromosomal anomalies conducted in our tertiary center. Material and Methods: A retrospective study was conducted to collect the results of prenatal invasive procedures performed in our tertiary center between January 2002-September 2017. The types and prevalence of fetal chromosomal anomalies were identified and the efficiency of the invasive procedure indications in predicting these fetal chromosomal anomalies was investigated. Results: The results of 2136 invasive procedures (2014 amniocentesis, 80 cordocentesis, and 42 chorion villus samplings) revealed $101(4.72 \%)$ fetal chromosomal anomalies and 128 (5.99\%) chromosomal polymorphisms. The most common chromosomal anomaly observed was trisomy 21 ( $n=52)$, followed by trisomy $18(n=12)$. In terms of diagnosis of the fetal chromosomal anomalies, the following observations were made: the indication "major structural anomaly in the ultrasound (USG)" exhibited 31.68\% sensitivity and $82.03 \%$ specificity with an odds ratio (OR) of 2.11 (95\% CI: 1.37-3.27); the indication "screening test positivity" exhibited $44.55 \%$ sensitivity and $48.25 \%$ specificity [OR=0.75 (95\% CI: $0.50-1.13$ )]; and the co-existence of "major structural anomaly in the USG" and "screening test positivity" exhibited 8.91\% sensitivity and $98.24 \%$ specificity [OR=5.49 (95\% CI: 2.54-11.84)]. Conclusion: Fetal chromosomal anomaly rate was observed to be $4.72 \%$. The co-existence of a sonographic major fetal structural anomaly and serum screening test positivity was observed to maximize the risk of numerical as well as a structural chromosomal anomaly. The data from the present study which reflected screening performances of detailed USG and biochemical screening tests for the detection of fetal chromosomal anomalies may be useful for clinicians while performing detailed counseling of patients.
\end{abstract}

Keywords: Amniocentesis; chromosome aberrations; fetus; prenatal ultrasonography

renatal diagnosis is crucial for providing the parents with an option to terminate the pregnancy, planning treatment methods post birth, and counseling the subsequent pregnancy. The screening and diagnosis of fetal aneuploidy in pregnancies may be performed using non-invasive as well as invasive procedures. The non-invasive screening tests include the first trimester combined tests (maternal age, serum free beta-human chorionic gonadotropin, pregnancy-associated plasma protein-A, and fetal nuchal translucency), the second trimester screening tests (alpha-fetoprotein, human chorionic gonadotropin, and estriol and/or inhibin A), and the analysis of cell-free DNA in the maternal blood, a method which has become widespread nowadays. ${ }^{1,2}$

The invasive procedures for prenatal diagnosis include amniocentesis, chorionic villus sampling (CVS), and cordocentesis. CVS and amniocentesis are performed at $11^{\text {th }}-14^{\text {th }}$ and $15^{\text {th }}-22^{\text {nd }}$ weeks of gestation, respectively. Cor- 
docentesis is performed at more advanced weeks of gestation. ${ }^{3}$ Among the aforementioned invasive procedures, CVS is a valuable diagnostic method as it allows diagnosis in early weeks of gestation, and also, it is possible to perform chromosomal analysis post this procedure through direct evaluation of the metaphase-stage cells present in the sample. ${ }^{4}$ The invasive procedure indications include a previous history of pregnancy with the chromosomal anomaly, abnormal prenatal screening test results, abnormal findings in ultrasonography (USG), and previously diagnosed maternal or paternal chromosomal anomalies. ${ }^{5}$ Subsequent to the detection of a risk factor for the fetal chromosomal anomaly, detailed counseling is required. Genetic counseling provides a clear understanding of prior risk assessment for fetal genetic pathology and fetal outcome, which allow the parents an informed choice regarding the invasive prenatal tests. ${ }^{6}$

Developments in the USG technology have enabled better diagnosis of the fetal structural anomalies and soft markers associated with chromosomal anomalies in the early weeks of gestation. ${ }^{7,8}$ It has been reported that the combination of the first and second trimester detailed USG evaluation was able to identify the major structural anomalies with a detection rate of $95 \%$. In congruence with the advancements in the USG technology, the developments in the fields of cytogenetics and molecular biology have provided an understanding of the genetic basis of several of these structural anomalies. Improvements in prenatal diagnostic technologies have been followed by the requirement of a detailed explanation of the advantages and disadvantages of the novel techniques. ${ }^{9}$ For example, chromosomal microarray technique is able to identify deletions or duplications in $1.7 \%$ of the cases with positive genetic screening who otherwise exhibit a normal classic karyotype. Therefore, it is important to recommend and use the most appropriate diagnostic test to detect a fetal genetic pathology. ${ }^{6}$

Faculty of Medicine, Trakya University, is the only center that provides the option of performing invasive prenatal tests in the Thrace Region. The present study was aimed at identifying the preva- lence of fetal chromosomal anomalies in the Thrace region by evaluating the results of invasive prenatal tests performed in our clinic between January, 2002 and September, 2017, as well as identifying the correlation between the diagnosed fetal chromosomal anomalies and the invasive prenatal test indications. The present study was designed as a retrospective investigation in order to present the regional data and evaluate and improve the knowledge regarding the matter from this perspective. The data from the present study may be able to assist the clinicians in providing detailed counseling to the prospective patients detected with a risk of fetal chromosomal anomaly regarding making a choice for or against the invasive procedures. Additionally, the findings of the present study may contribute to reducing anxiety in such parents.

\section{MATERIAL AND METHODS}

The present retrospective study evaluated the results of invasive prenatal tests performed at the Department of Obstetrics and Gynecology, Faculty of Medicine, Trakya University, between January 2002 and September 2017. Ethical approval for the study was obtained from the Ethics Committee, Trakya University (Ethics Committee protocol code: 2017/307, 2017). The study was conducted in accordance with the Helsinki declaration.

The results of 2136 invasive prenatal tests were evaluated, and pregnant women whose invasive procedure results indicated numerical or structural fetal chromosomal anomalies were identified. The fetal chromosomal anomaly rate among pregnant women who underwent invasive procedures in our center was calculated. The invasive procedure indications included a high risk identified in screening tests (threshold values: $1 / 270$ for the first trimester combined and quad test, and 1/300 for the triple test), and a minimum of two soft markers or one major structural anomaly identified in USG. Soft markers in the fetus included a short femur/humerus, choroid plexus cyst, echogenic cardiac focus, hyperechogenic bowel, renal pyelectasis, hypoplastic nasal bone, and single umbilical artery. Major structural anomalies included serious defects, such as omphalocele, cystic hy- 
groma, megacystis, renal agenesis, extremity anomalies except the isolated short humerus/femur condition, major cardiac anomalies (ventricular septal defect, atrioventricular septal defect, cardiac output and four chamber anomalies, etc.), and intracranial anomalies (holoprosencephaly, ventriculomegaly, posterior fossa anomalies, etc.). In addition to the aforementioned indications, a few indications such as advanced maternal age (AMA), previous history of pregnancy with the chromosomal anomaly, maternal or paternal chromosomal diseases, and maternal anxiety were categorized as "other indications". The patients who refused the recommended invasive procedures, the pregnancies that were terminated without invasive procedures due to complex major anomalies, and the pregnancies for which no records could be obtained were excluded from the present study.

In the present study, fetal USG was performed at $11^{\text {th }}-14^{\text {th }}$ weeks and at $18^{\text {th }}-22^{\text {nd }}$ weeks of gestation. The USG was performed using Voluson 730 Expert (GE Healthcare, Milwaukee, WI, USA) and Voluson E6 (GE Healthcare, Zipf, Austria) USG equipment. CVS was performed at $11^{\text {th }}-14^{\text {th }}$ weeks of gestation by performing aspiration of the chorionic villous tissue from a suitable placental area using a 20-gauge CVS needle. Amniocentesis was performed at $16^{\text {th }}-22^{\text {nd }}$ weeks of gestation using a 22-gauge spinal needle. In the amniocentesis, the procedure, $2 \mathrm{~mL}$ of amniotic fluid was taken out initially and removed, as this fluid has maternal contamination. Subsequently, $20 \mathrm{~mL}$ of amniotic fluid was obtained for genetic analysis. Cordocentesis was performed using an 18-gauge spinal needle for the aspiration of approximately $3 \mathrm{~mL}$ of blood from the umbilical vein present at the location where the fetal cord enters placenta. Fetal blood was placed in heparin-containing vacutainers and sent to the genetics laboratory. The skin of the parents undergoing the tests was disinfected with $10 \%$ povidone-iodine prior to all the invasive procedures. Prior to cordocentesis and CVS procedures, local anesthesia was applied to the abdominal skin along with lidocaine. The cases with $\mathrm{Rh}$ incompatibility were given $300 \mathrm{mcg}$ Anti-D IgG post the procedure. All the patients were hospital- ized for at least $2 \mathrm{~h}$ post each invasive procedure and then discharged. The genetic analysis was performed with a minimum of 500-550 resolutions using the Giemsa trypsin banding method on at least 20 metaphase plates. The cell culture results were obtained within approximately 20-25 days.

SPSS version 20.0 (IBM, Armonk, NY, USA) program was utilized for statistical analyses. While evaluating the study data, descriptive statistical methods, such as ratio, sensitivity, specificity, positive and negative predictive values, and false positive and negative rates, were used. Pearson's Chi-Squared test and Fisher's Exact test were used to compare qualitative data. Odds ratios for each invasive procedure indication were calculated. The results were evaluated in 95\% confidence interval at a significance level of $\mathrm{p}<0.05$.

\section{RESULTS}

The present study evaluated the test results of 2136 invasive procedures, which included 2014 amniocentesis, 80 cordocentesis, and 42 CVS. The average maternal ages observed in the amniocentesis, cordocentesis, and CVS groups were 27.55 \pm 3.74 years, $28.12 \pm 4.37$ years, and $26.29 \pm 5.59$ years, respectively. The average gestational weeks at the time of the invasive procedure in the amniocentesis, cordocentesis, and CVS groups were $18.64 \pm 1.36$ weeks, $23.36 \pm 0.95$ weeks, and $12.44 \pm 0.79$ weeks, respectively. In the present study, $2.76 \%$ of the invasive procedures $(n=59)$ exhibited no growth in the sample culture, and $1.02 \%$ of the procedures $(\mathrm{n}=22)$ exhibited maternal contamination (Table 1).

The results revealed 101 fetal chromosomal anomalies (4.72\%) and 128 fetal chromosomal polymorphisms (5.99\%). The most common chromosomal anomaly was trisomy $21(\mathrm{n}=52)$, followed by trisomy $18(\mathrm{n}=12)$. The highest chromosomal anomaly rates were observed in the CVS results $(33.33 \%, \mathrm{n}=14)$. All the chromosomal anomalies identified in the results are presented in Table 1 along with their distributions.

In the evaluation of the invasive procedure indications, it was observed that in case of co-ex- 


\begin{tabular}{|c|c|c|c|c|}
\hline & Amniocentesis ( $n=2.014)$ & Cordocentesis $(n=80)$ & Chorion villus sampling ( $n=42)$ & Total $(n=2136)$ \\
\hline Maternal age (mean $\pm S D)$ & $27.55 \pm 3.74$ & $28.12 \pm 4.37$ & $26.29 \pm 5.59$ & \\
\hline Gestational age (weeks) $\left(\right.$ mean $\left._{ \pm} S D\right)$ & $18.64 \pm 1.36$ & $23.36 \pm 0.95$ & $12.44 \pm 0.79$ & \\
\hline Trisomy 21 (n) & 45 & 4 & 3 & 52 \\
\hline Trisomy 18 (n) & 10 & 0 & 2 & 12 \\
\hline Trisomy 13 (n) & 1 & 0 & 3 & 4 \\
\hline Turner syndrome (n) & 4 & 0 & 3 & 7 \\
\hline Mosaic turner (n) & 1 & 0 & 1 & 2 \\
\hline Mosaic trisomy 18 (n) & 1 & 0 & 0 & 1 \\
\hline Klinefelter syndrome ( $\mathrm{n}$ ) & 2 & 0 & 0 & 2 \\
\hline Chromosomal polymorphism (n) & 118 & 7 & 3 & 128 \\
\hline Triploidy (n) & 1 & 0 & 0 & 1 \\
\hline Tetraploidy (n) & 1 & 0 & 0 & 1 \\
\hline \multirow[t]{7}{*}{ Reciprocal translocation } & $46,--, t(13 ; 20)(q 12 ; p 11.2)$ & & $46,--, t(8 ; 9)(q 23.3 ; p 24.2)$ & \\
\hline & 46,XX,t (2;6)(q33;p23) & & $46,--, t(13 ; 20)(q 10 ; p 10)$ & \\
\hline & $46,-, t(3 ; 5)(q 29 ; p 12)$ & & & \\
\hline & $46,--, t(2 ; 15)(q 35 ; q 22.3)$ & & & \\
\hline & $46,--, t(X ; 12)(q 23 ; q 25)$ & & & \\
\hline & $46, X X, t(5 ; 15)(p 13 ; q 11.2)$ & & & \\
\hline & 46,--,t (5;11)(q31;p13) & & & \\
\hline Robertsonian translocation & $46,--, \operatorname{rob}(14 ; 21)(q 10 ; q 10)$ & & & \\
\hline \multirow[t]{9}{*}{ Others } & $46, X^{\star}$, del $(5)(q 15)[2] / 46, x^{*}[28]$ için düzey I & I mozaiklik & & \\
\hline & 46,--dup (9)(p11p13) & & & \\
\hline & $46,--\operatorname{der}(17) t(3 ; 17)(\mathrm{p} 22 ; \mathrm{p} 13)$ & & & \\
\hline & $48, X X Y Y$ & & & \\
\hline & 47,--,+mar[2], 46,--[58] & & & \\
\hline & $46,--(48) / 47,--,+\operatorname{mar}(2)$ & & & \\
\hline & $46,--$, del $(18)(p 11.2)[1 / 20] / 46,--[19 /$ & & & \\
\hline & 46,XY,t (3;5;8)(p23;p15?;q21?) & & & \\
\hline & $47, x^{*}, \operatorname{der}(18) t(X: 18)(q 11 ; q 11),+m$ & & & \\
\hline Non-growth samples in culture (n) & 35 & 11 & 13 & 59 \\
\hline Maternal contamination ( $\mathrm{n}$ ) & 14 & 5 & 3 & 22 \\
\hline Total chromosomal anomalies (n,\%) & $83(4.12 \%)$ & $4(5 \%)$ & $14(33.33 \%)$ & $101(4.72 \%)$ \\
\hline
\end{tabular}

SD: Standard deviation.

istence of "major structural anomaly in the USG" and "screening test positivity", $21.95 \%$ of the cases $(\mathrm{n}=9)$ presented fetal chromosomal anomaly, while in case of indication "major structural anomaly in the USG" alone, $8.88 \%$ of the cases $(\mathrm{n}=32)$ presented fetal chromosomal anomaly (Table 2). In terms of diagnosis of the fetal chromosomal anomalies, the indication "major structural anomaly in the USG" demonstrated 31.68\% sensitivity and $82.03 \%$ specificity, the indication "screening test positivity" demonstrated $44.55 \%$ sensitivity and $48.25 \%$ specificity, and the co-existence of "major structural anomaly in the USG" and "screening test positivity" demonstrated 8.91\% sensitivity and $98.24 \%$ specificity (Table 3 ). The highest odds ratio (OR) of 5.49 (95\% CI: 2.5411.84) observed in the diagnosis of chromosomal anomaly was obtained with the co-existence of "major structural anomaly in the USG" and "screening test positivity", followed by "major structural anomaly in the USG" with an OR of: 2.11 (95\% CI: 1.37-3.27) (Table 4). 


\begin{tabular}{|lccc|}
\hline \multicolumn{4}{|c|}{ TABLE 2: The number of fetal chromosomal anomalies according to invasive procedure indications. } \\
\hline Invasive test indications & Chromosomal anomalies $(\mathbf{n})$ & Total patients $(\mathbf{n})$ & Chromosomal anomaly rates (\%) \\
\hline Major structural anomaly in the USG & 32 & 360 & 8.88 \\
Screening test positivity & 45 & 985 & 4.56 \\
Major structural anomaly in the USG+screening test positivity & 9 & 41 & 21.95 \\
Soft marker in the USG & 4 & 148 & 2.7 \\
Soft marker in the USG+Screening test positivity & 5 & 31 & 1.04 \\
Other indications & 6 & 322 & 1.86 \\
\hline
\end{tabular}

USG: Ultrasonography.

\begin{tabular}{|lcccccc|}
\hline \multicolumn{7}{|c|}{ TABLE 3: } \\
\hline Invasive test indications & Sensitivity & Specificity & PPV (\%) & NPV (\%) & False positive rate (\%) & False negative rate (\%) \\
\hline Major structural anomaly in the USG & 31.68 & 82.03 & 8.88 & 95.59 & 17.96 & 68.31 \\
\hline Screening test positivity & 44.55 & 48.25 & 4.56 & 94.05 & 51.75 & 55.44 \\
Major structural anomaly in the USG+screening test positivity & 8.91 & 98.24 & 21.95 & 95.12 & 1.75 & 91.08 \\
\hline Soft marker in the USG & 3.96 & 92.11 & 2.7 & 94.54 & 7.88 & 96.03 \\
Soft marker in the USG+screening test positivity & 4.95 & 96.38 & 7.04 & 94.82 & 3.61 & 95.04 \\
\hline
\end{tabular}

PPV: Positive predictive value; NPV: Negative predictive value; USG: Ultrasonography.

\begin{tabular}{|c|c|c|c|c|c|}
\hline & Chromosomal anomaly (+) & Chromosomal anomaly (-) & & & \\
\hline Invasive test indications & $(n, \%)$ & $(n, \%)$ & Total patients $(\mathrm{n})$ & $\mathrm{p}$ & ODDS $(95 \% \mathrm{Cl})$ \\
\hline Major structural anomaly in the USG & $32(31.6)$ & $328(18)$ & 360 & $0.001^{* *}$ & $2.11(1.37-3.27)$ \\
\hline Screening test positivity & $45(44.5)$ & $940(51.47)$ & 985 & 0.17 & $0.75(0.50-1.13)$ \\
\hline Major structural anomaly in the USG+screening test positivity & $9(8.9)$ & $32(1.7)$ & 41 & $0.001^{* *}$ & $5.49(2.54-11.84)$ \\
\hline Soft marker in the USG & $4(4.0)$ & $144(7.9)$ & 148 & 0.149 & $0.48(0.17-1.32)$ \\
\hline Soft marker in the USG+Screening test positivity & $5(5.0)$ & $66(3.7)$ & 71 & 0.417 & $1.38(0.54-3.52)$ \\
\hline Other indications & $6(5.9)$ & $316(17.3)$ & 322 & 0.273 & $0.62(0.27-1.45)$ \\
\hline Total $(n)$ & 101 & 1.826 & 1.927 & & \\
\hline
\end{tabular}

USG: Ultrasonography; Cl: Confidence interval; ${ }^{* \star} p<0.01$.

\section{DISCUSSION}

The present study evaluated the fetal chromosomal anomaly rate in the Thrace Region of Turkey and the correlation between the fetal chromosomal anomalies and the invasive prenatal test indications. The fetal chromosomal anomaly rate was observed to be $4.72 \%$, and the most common fetal chromosomal anomaly identified for the Thrace Region was trisomy 21. The combination of "sonographic major structural fetal anomaly" and "serum screening test positivity" indications for the prenatal invasive procedure demonstrated the highest odds ratio in the diagnosis of fetal chromosomal anomaly.

The widespread use of screening tests and detailed USG examinations has led to an increase in the number of invasive procedures performed. ${ }^{10}$ Various studies in Turkey have revealed that 3.3\%$4.98 \%$ of the pregnant women who underwent invasive procedures received a diagnosis for fetal chromosomal anomalies. ${ }^{11-13}$ Since our institution is the only tertiary center that performs invasive prenatal tests in the Thrace Region, the data from the present study reflect regional statistics. The present study revealed that the Thrace region had 
a fetal chromosomal anomaly rate of $4.72 \%$, which was similar to the fetal chromosomal anomaly rates reported from the other regional centers. ${ }^{11-13}$ The reason for obtaining a similar rate could be the recent migrations, as it is known that the Thrace Region has lower inbreeding and pregnancy rates compared to the other regions in the country. Moreover, the rates of chromosomal polymorphism, described as the variations at the chromosomal regions known as heterochromatin, obtained in the present study (5.99\%) were also observed to be consistent with those reported in the literature. $^{14}$

The first trimester combined test including nuchal translucency and biochemical parameters was able to detect $90 \%$ of all the trisomy 21 cases, with a false positive rate of $5 \%$. If this test was enhanced by combining it with the other USG parameters (nasal bone, ductus venosus, and tricuspid regurgitation), the detection rate was observed to improve and reached $91 \%-96 \% .{ }^{15}$ In the present study, the highest fetal chromosomal anomaly rate was obtained in pregnant women who underwent the CVS (33.33\%) procedure; this finding was also consistent with the literature. ${ }^{4}$ It is, therefore, proposed that the first-trimester screening tests are efficient in diagnosing fetal aneuploidy and that the major structural anomalies that could be diagnosed in the first trimester are more related to the chromosomal anomalies. Therefore, it would be more convenient to use detailed USG along with the combined test to detect fetal aneuploidy in the first trimester, as stated in the literature as well. ${ }^{15}$

It has been reported previously in the literature that the most common indications for amniocentesis were high risk in triple screening test, AMA, and positive USG findings, and that the most common fetal chromosomal anomaly observed was trisomy $21 .{ }^{12,13}$ The present study revealed screening test positivity and major structural anomaly in the USG as the most common invasive procedure indications, while the most common fetal chromosomal anomaly revealed in the present study was trisomy 21. In this context, the results of the present study were consistent with the literature. In the present study, AMA, maternal anxiety, history of pregnancy with the chromosomal anomaly, and maternal or paternal chromosomal diseases were categorized as "other indications". AMA has been rejected as an invasive procedure indication by itself in several centers recently. ${ }^{16}$ Our clinic adopted this approach, and lately, AMA has been accepted as a parameter to be used in calculating the risk of aneuploidy in the screening tests only. Therefore, in the present study, AMA was not evaluated as a separate invasive procedure indication. In the present study, $2.76 \%$ of the cases presented no growth in the cell culture, and $1.02 \%$ of the cases presented maternal contamination; these rates were lower than the rates reported in the literature. ${ }^{12,13}$ As our own laboratories began providing the facility to perform genetic analyses, these rates were observed to decrease in comparison to the rates obtained during the period when such services were provided by external laboratories. It was, therefore, concluded that differences in the environmental conditions and equipment of the facilities where these procedures were performed, the experience of the staff, and the material transport conditions may have affected the culture success.

A study by Saatçi et al. reported that abnormal fetal karyotype was detected in $4.1 \%$ of the pregnant women who obtained abnormal screening test results. ${ }^{13}$ Another study by Yüce et al. identified fetal chromosomal anomalies in $3.7 \%$ of the pregnant women who underwent invasive procedures due to high risk-indicating results obtained in the triple screening test. ${ }^{11}$ In the present study as well, fetal chromosomal anomalies were identified in $4.56 \%$ of the cases that had received positive screening test results. In this context, the results of the present study were close to the ones reported in the literature. Moreover, when the aforementioned results of the present study were re-analyzed in combination with the USG findings (soft markers or major structural fetal anomalies), lower rates were observed. It was, therefore, concluded that the coexistence of abnormal screening test results and USG findings increased the risk of fetal chromosomal anomaly. Supporting the soft markers with screening tests is important for the prediction of fetal chromosomal anomalies. 
Major congenital anomalies occur in approximately $5 \%$ of live births. Most of these anomalies have been reported to be detected in the prenatal period. ${ }^{17}$ Detailed USG examination aids in determining the underlying genetic etiology of such anomalies. ${ }^{6}$ Obstetric USG is used, either alone or in combination with other screening tests, for the screening of fetal chromosomal anomaly. ${ }^{8}$ It was reported that the absence of any USG findings related to Down's syndrome decreased the risk of the chromosomal anomaly by $60-80 \%$ and that $75 \%$ of the fetuses with Down's syndrome received USG findings. ${ }^{18}$ Furthermore, the rate of false-positives for Down's syndrome was reported to decrease when the second-trimester screening tests were performed in combination with obstetric USG. ${ }^{8}$ In the present study, consistent with the literature, the rate of false-positives obtained with the co-existence of "major structural anomaly in the USG" and "screening test positivity" was observed to be lower compared to the cases in which the aforementioned indications were present alone. A strong association between prenatal USG findings and fetal chromosomal anomalies has been reported by previous studies. ${ }^{19,20}$ A study by Saatçi et al. identified fetal chromosomal anomalies in $10.84 \%$ of the cases that received abnormal USG findings. ${ }^{13}$ In the present study, consistent with the literature, $8.88 \%$ of the cases with a major structural anomaly in the USG presented fetal chromosomal anomaly, and this rate increased to $21.95 \%$ when the screening test positivity co-existed with the structural anomaly. In addition to major congenital anomalies, the screening test positivity was observed to increase the risk of the fetal chromosomal anomaly by more than two times. This significant increase revealed a synergistic effect of the screening tests on the occurrence of a chromosomal anomaly. This finding may be useful in the detailed counseling of prospective parents who do not desire to undergo any invasive procedures despite the presence of screening test positivity in addition to the congenital major anomaly. Post counseling and explanations, such parents might reconsider their negative attitude toward invasive procedures. In addition, the highest odds ratio in the diagnosis of a fetal chromosomal anomaly as obtained in the present study was 5.49 (95\% CI: 2.54-11.84), and it was obtained in the cases of combination of "screening test positivity" and "major structural anomaly in the USG" indications. The odds ratio value obtained for the "screening test positivity" indication was not statistically significant. The reason underlying this finding could be that in addition to trisomies, other numerical and structural chromosomal anomalies have also been evaluated in the present study. An overall evaluation of the aforementioned findings of the present study indicated that, for the pregnant women with screening test positivity, a detailed fetal USG examination could be able to predict possible chromosomal anomalies. A previous study reported that in fetuses for which an increased nuchal translucency was detected in the first trimester, the odds ratio for observing a structural fetal anomaly in the diagnosis of fetal chromosomal anomaly was 8.15, which was higher than the odds ratio obtained in the results of the present study; the reason for this could be that all the cases in that study demonstrated increased nuchal translucency, unlike the cases in the present study. ${ }^{21}$

While the existence of sonographic soft markers has been reported to increase the risk of fetal aneuploidy, the lack of a standardized definition for soft markers and the subjective descriptions lead to critical variations in the aneuploidy detection rates. ${ }^{7}$ Benacerraf et al., in their study, examined a pregnant women who had been implemented with amniocentesis, and had fetuses with short femur and humerus. ${ }^{22}$ The authors observed that in the group where the risk of trisomy was $1 / 250$, these USG markers had a positive predictive value of $4.56 \%$ in the diagnosis of trisomy, and in the group where the risk was $1 / 1000$, PPV was $1.18 \% .{ }^{22}$ Similar to the aforementioned study, it was observed in the present study that, in the diagnosis of chromosomal anomaly, the PPV for the combination of "soft marker in the USG" and "screening test positivity" indications was higher than the PPV for the soft marker indication alone. Additionally, PPVs obtained in the present study were higher than the values reported in the litera- 
ture. This difference could have risen as that study used only maternal age and maternal serum alphafetoprotein values in the calculation of risk of trisomy, while the present study utilized all the results of the first and second-trimester screening tests in addition to examining the shortness of extremities along with the evaluation of all soft markers. ${ }^{22}$ In the present study, soft marker positivity demonstrated a lower sensitivity value compared to those reported in the literature, which could have occurred because soft markers are generally known to be associated with Down's syndrome, while in the present study, the sensitivity values were calculated for all the numerical and structural chromosomal anomalies..$^{22}$ Moreover, in the present study, the co-existence of "soft markers" and "screening test positivity" demonstrated higher sensitivity and specificity values compared to the soft marker and positivity indications when alone. This finding demonstrated that combining the sonographic soft markers with screening tests could serve as a better method for diagnosing aneuploidy.

Prenatal counseling begins with discussing patient's chronic diseases, obstetric history, and personal and family characteristics, and has been associated with reduced physiological distress. ${ }^{6,17}$ Prenatal diagnosis of a fetal anomaly causes emotional stress, sadness, and fear among prospective parents. ${ }^{17}$ It is, therefore, essential to provide information to the prospective parents regarding the possible risks, post the evaluation of all risks. Clinicians should counsel pregnant women with a high risk of a fetal genetic anomaly in order to ensure that the prospective parents have a clear understanding of the possible risks involved. Prenatal screening models, including the biochemical and/or USG evaluation of risks, have been reported to be useful in diagnosing chromosomal anomalies. ${ }^{6}$ The present study evaluated the relationship between invasive test indications and the results of the fetal chromosomal analysis. Among all the prenatal test indications, the combination of "major fetal anomaly" and "screening test positivity" presented the highest rate of fetal chromosomal anomaly. The rates of chromosomal anomalies for other invasive test indications were also reported in the present study. The findings of the present study may be useful for clinicians in providing detailed counseling to prospective parents as the study provides concrete information regarding the estimated risk of a fetal chromosomal anomaly, which may contribute to reducing anxiety in such parents.

The limitation of the present study was that the investigation of the relationship between chromosomal anomalies and the invasive test indications was performed as a whole without discriminating on the basis of types of chromosomal anomalies. Therefore, the importance of prenatal invasive test indications in the diagnosis of trisomies or other chromosomal abnormalities could not be evaluated separately. The strength of the present study was that the study has reported invasive prenatal test results and prevalence of fetal chromosomal abnormalities in the Thrace Region of Turkey, and also, that it evaluated the efficiency of the invasive test indications in predicting fetal chromosomal anomalies.

\section{CONCLUSION}

Fetal chromosomal anomaly rate in the Thrace Region of Turkey as obtained in the present study was $4.72 \%$, which is close to the rates reported in the other centers of the country. This finding could be useful for establishing regional data, and for comparing these data with the chromosomal anomaly rates in other regions. Detailed USG is crucial for the screening of fetal chromosomal anomalies. Pregnant women presenting the combination of "major structural fetal anomaly" and "screening test positivity" indications constituted the group of highest risk in terms of chromosomal anomalies. Evaluating the maternal serum-screening test results in combination with the USG findings increased the rate of diagnosis of possible fetal chromosomal anomalies. Prenatal test indications serve as important directive factors in the prediction of fetal chromosomal anomalies. The results of the present study may be useful in determining predictive risks for chromosomal anomalies. Therefore, these findings may contribute in reducing anxiety and assist them in taking the right decision regarding the invasive procedures. 


\section{Source of Finance}

During this study, no financial or spiritual support was received neither from any pharmaceutical company that has a direct connection with the research subject, nor from a company that provides or produces medical instruments and materials which may negatively affect the evaluation process of this study.

\section{Conflict of Interest}

No conflicts of interest between the authors and / or family members of the scientific and medical committee members or members of the potential conflicts of interest, counseling, ex- pertise, working conditions, share holding and similar situations in any firm.

\section{Authorship Contributions}

Concept: All authors; Design: Cihan İnan, N. Cenk Sayın, Hakan Gürkan, Z. Nihal Dolgun, Selen Gürsoy Erzincan, Işıl Uzun, Havva Sütçü; Supervision: All authors; Data collection: Cihan İnan, N. Cenk Sayın, Hakan Gürkan; Analysis and literature review: Cihan İnan, N. Cenk Sayın, Z. Nihal Dolgun, Füsun Varol; Writing the article: Cihan İnan, N. Cenk Sayın, Z. Nihal Dolgun; Critical Review: Cihan İnan, N. Cenk Sayın, Z. Nihal Dolgun, Füsun Varol. References and fundings: Cihan İnan.

\section{REFERENCES}

1. Palomaki GE, Kloza EM, Lambert-Messerlian GM, Haddow JE, Neveux LM, Ehrich M, et al. DNA sequencing of maternal plasma to detect Down syndrome: an international clinical validation study. Genet Med. 2011;13(11):91320. [Crossref] [PubMed]

2. Cicero $S$, Bindra R, Rembouskos $G$, Spencer $\mathrm{K}$, Nicolaides KH. Integrated ultrasound and biochemical screening for trisomy 21 using fetal nuchal translucency, absent fetal nasal home, free beta-hCG and PAPP-A at 11 to 14 weeks. Prenat Diagn. 2003;23(4):306-10. [Crossref] [PubMed]

3. Neagos D, Cretu R, Sfetea RC, Bohiltea LC. The importance of screening and prenatal diagnosis in the identification of the numerical chromosomal abnormalities. Maedica (Buchar). 2011;6(3):179-84.

4. Bilen E, Yüksel M, Köse SA, Tola EN, Sezik M. Chorion villus sampling for karyotyping at 11-14 weeks of gestation: evaluation of 42 cases. SDÜ Sağlık Bilimleri Dergisi. 2015;6(1): 1-3.

5. Farcaş S, Crişan CD, Andreescu N, Stoian M, Motoc AG. Structural chromosomal anomalies detected by prenatal genetic diagnosis: our experience. Rom J Morphol Embryol. 2013;54 (2):377-83. [PubMed]

6. Wilson RD, Gagnon A, Audibert F, Campagnolo C, Carroll J; Genetics Committee. Prenatal diagnosis procedures and techniques to obtain a diagnostic fetal specimen or tissue: maternal and fetal risks and benefits. J Obstet Gynaecol Can. 2015;37(7):656-68. [Crossref]

7. Norton ME. Follow-up of sonographically detected soft markers for fetal aneuploidy.
Semin Perinatol. 2013;37(5):365-9. [Crossref] [PubMed]

8. Anderson CL, Brown CE. Fetal chromosomal abnormalities: antenatal screening and diagnosis. Am Fam Physician. 2009;79(2):117-23. [PubMed]

9. Levy B, Stosic M. Traditional prenatal diagnosis: past to present. Methods Mol Biol. 2019;1885:3-22. [Crossref] [PubMed]

10. Ekmekci E, Kurt K, Gençdal S, Demirel E, Kelekçi S. Prenatal invasive testing: a 4-years single institution experience in Turkey. Gynecol Obstet Reprod Med. 2015;21(3):123-6.

11. Yüce $H$, Çelik $H$, Gürateş $B$, Erol $D$, Hanay $F$, Elyas $\mathrm{H}$. [Retrospective analysis of $356 \mathrm{am}$ niocentesis results performed for karyotype analysis]. Perinatoloji Dergisi. 2006;14(2):736.

12. Erdemoğlu M, Kale A. Prospective analysis of 183 cases who under go for amniocentesis of genetic karyotyping. Dicle Tip Dergisi. 2007;34(3):170-5.

13. Saatçi Ç, Bayramov R, Basbuğ M, Güneş MC, Dündar M. Retrospective evaluation of results of 3617 invasive prenatal diagnosis cases applied between 1997-2015 years. Journal of Health Sciences. 2016;25(3):120-5.

14. Mierla D, Stoian V. Chromosomal polymorphisms involved in reproductive failure in the Romanian population. Balkan J Med Genet. 2012;15(2):23-8. [Crossref] [PubMed] [PMC]

15. Nemescu D, Bratie A, Mihaila A, Navolan D, Tanase A. First trimester combined screening for fetal aneuploidies enhanced with additional ultrasound markers: an 8-year prospective study. Ginekol Pol. 2018;89(4):205-10. [Crossref] [PubMed]

16. Godino L, Pompilii E, D'Anna F, Morselli-Labate AM, Nardi E, Seri M, et al. Attitudes of women of advanced maternal age undergoing invasive prenatal diagnosis and the impact of genetic counselling. Eur J Hum Genet. 2016;24(3):331-7. [Crossref] [PubMed] [PMC]

17. Marokakis S, Kasparian NA, Kennedy SE. Prenatal counselling for congenital anomalies: a systematic review. Prenat Diagn. 2016;36 (7):662-71. [Crossref] [PubMed]

18. Benacerraf BR. The role of the second trimester genetic sonogram in screening for fetal Down syndrome. Semin Perinatol. 2005; 29(6):386-94. [Crossref] [PubMed]

19. Stoll C, Dott B, Alembik Y, Roth MP. Evalution of routine prenatal ultrasound examination in detecting fetal chromosomal abnormalities in a low risk population. Hum Genet. 1993;91(1): 37-41. [Crossref] [PubMed]

20. Conner SN, Longman RE, Cahill AG. The role of ultrasound in the diagnosis of fetal genetic syndromes. Best Pract Res Clin Obstet Gynaecol. 2014;28(3):417-28. [Crossref] [PubMed] [PMC]

21. Zalel $Y$, Zemet R, Kivilevitch $Z$. The added value of detailed early anomaly scan in fetuses with increased nuchal translucency. Prenat Diagn. 2017;37(3):235-43. [Crossref] [PubMed]

22. Benacerraf BR, Neuberg D, Bromley B, Frigoletto FD Jr. Sonographic scoring index for prenatal detection of chromosomal abnormalities. J Ultrasound Med. 1992;11(9):449-58. [Crossref] [PubMed] 